\title{
TWO REMARKS ON GLOBAL SOLUTIONS OF ORDINARY DIFFERENTIAL EQUATIONS IN THE REAL LINE
}

\author{
GIOVANNI VIDOSSICH
}

\begin{abstract}
We prove two theorems concerning global solutions of initial and terminal value problems: a criteria for the existence of maximal and minimal solutions, as well as the connectedness of the set of solutions without any assumption but the Carathéodory hypotheses.
\end{abstract}

During the preparation of [6] it appeared that on the real line, due to its rich structure, we can have stronger results concerning global solutions of the terminal value problem for ordinary differential equations. These results seem to be significant also for the Cauchy problem and, therefore, they are collected here for both problems. We prove two theorems concerning global solutions of initial and terminal value problems: (i) a necessary and sufficient condition for the existence of maximal and minimal solutions, and (ii) the set of solutions is always connected. These results are well known for local solutions, while for global solutions the connectedness of the set of solutions under some restrictive assumptions on the domain was proved very recently by Kelley [2] and Nakagiri-Murakami [3]. Moreover, it is well known that a maximal solution has a maximal interval of existence, and this is an almost global result. It may be interesting to notice that the proofs are based only on General Topology, avoiding the customary techniques of ordinary differential equations, and that perhaps for the first time the hypotheses of Carathéodory are used as an essential tool in proving a theorem (cf. the proof of Theorem 2).

The Cauchy problem is not considered here in the classical sense, but rather as a limit Cauchy problem in the sense that the starting point $\left(t_{0}, x_{0}\right)$ is assumed to be only in the closure of the domain of the function in the second member of the given equation. This setting permits us to solve a terminal value problem as a suitable Cauchy problem by simply performing a change of variable. The example $A=] 0,1], f(x)=\sin x^{-1}$ shows that this setting is not always equivalent to the classical one even under Carathéodory hypotheses. Therefore, Theorem 2 below is not included in the results of Kelley [2] and Nakagiri-Murakami [3].

0. Preliminaries. Recall that the generalized Carathéodory hypotheses on a function $f: I \times A \rightarrow \mathbf{R}, I$ an interval and $A$ any subset of $\mathbf{R}$, are the following:

Received by the editors July 29, 1974 and, in revised form, August 19, 1974 and December 26, 1974.

AMS (MOS) subject classifications (1970). Primary 34A15.

Key words and phrases. Cauchy problem, terminal value problem, global solution, maximal and minimal solution, connectedness of the set of solutions, Carathéodory hypotheses. 
$\left(\mathrm{C}_{1}\right) f(\cdot, x): I \rightarrow \mathbf{R}$ is measurable for all $x \in A$;

$\left(\mathrm{C}_{2}\right) f(t, \cdot): A \rightarrow \mathbf{R}$ is continuous for a.e. $t \in I$;

$\left(\mathrm{C}_{3}\right)$ For every bounded set $B \subseteq A$ there exists $h_{B} \in L_{\mathrm{loc}}^{1}\left(I, \mathbf{R}^{+}\right)$such that

$$
\left.|f(t, x)| \leqq h_{B}(t) \quad \text { (a.e. } t \in I, x \in B\right) \quad \text { and } \quad \int_{I} h_{B}(s) d s<+\infty ;
$$

where $\int_{I} h(s) d s$ means the improper integral when $I$ is not compact (we are interested in the cases $I=] a, b]$ and $I=\left[a,+\infty[)\right.$. By solution of $x^{\prime}=f(t, x)$ we mean a function $x$ which is absolutely continuous on compact intervals and satisfies the given equation almost everywhere.

The following lemma is well known, but we prove it for completeness since it is a crucial starting point in the proofs of our results.

Lemma. Let $I$ be any interval of $\mathbf{R}, A \subseteq \mathbf{R}$ and let $f: I \times A \rightarrow \mathbf{R}$ satisfy the generalized Carathéodory hypotheses. If $u$ and $v$ are solutions of $x^{\prime}=f(t, x)$, then $w=\max \{u, v\}$ is also.

Proof. Obviously, $w^{\prime}=f(t, w)$ a.e. where $v>u$ or $u<v$, as well as in the interior of $A=\{t \in I \mid u(t)=v(t)\}$ in $\mathbf{R}$. Let $t_{0}$ be a frontier point of $A$ in $\mathbf{R}$ where $u$ and $v$ satisfy the equation. Since $w\left(t_{0}\right)=u\left(t_{0}\right)$ and $u^{\prime}\left(t_{0}\right)=f\left(t, w\left(t_{0}\right)\right)$ $=v^{\prime}\left(t_{0}\right)$, for every $\varepsilon>0$ there is $\delta>0$ such that

$$
\begin{array}{r}
\left|\frac{u(t)-w\left(t_{0}\right)}{t-t_{0}}-f\left(t_{0}, w\left(t_{0}\right)\right)\right|<\varepsilon \text { and }\left|\frac{v(t)-w\left(t_{0}\right)}{t-t_{0}}-f\left(t_{0}, w\left(t_{0}\right)\right)\right| \\
(t \in \varepsilon \\
\left(t \in I ;-t_{0} \mid<\delta\right) .
\end{array}
$$

Substituting $w(t)$ for $u(t)$ where $u \geqq v$ and $w(t)$ for $v(t)$ where $v \geqq u$, we have $w^{\prime}\left(t_{0}\right)=f\left(t_{0}, w\left(t_{0}\right)\right)$. Finally, $w$ is Lipschitz on compact intervals since $w$ $=\frac{1}{2}(u+v+|u-v|)$. Therefore $w$ is absolutely continuous on compact intervals of $I$. Q.E.D.

1. A necessary and sufficient condition for the existence of maximal and minimal solutions.

Theorem 1. Let $A \subseteq \mathbf{R}$ be closed and let $f:] a, b] \times A \rightarrow \mathbf{R}$ satisfy the generalized Carathéodory hypotheses. For the limit Cauchy problem

$$
x^{\prime}=f(t, x), \quad \lim _{t \downarrow a} x(t)=x_{0},
$$

there exists the maximal (resp., the minimal) solution in $] a, b]$ if and only if there exists at least one solution in $] a, b]$ and all solutions are bounded above (resp., below) by a constant $c$.

Proof. We prove only the statement on the maximal solution since the other proof is similar. If the maximal solution $u_{M}$ exists, then $u_{M}$ has a continuous extension $\bar{u}_{M}$ on $[a, b]$, and it is enough to take $c=\max \bar{u}_{M}$ in order to have the necessity. Conversely, assume the set $S$ of solutions of our problem is bounded above by a constant $c$. Each member of $S$ has a continuous extension to $[a, b]$, and, therefore, we consider $S$ as a subset of $C([a, b])$, the space of real-valued continuous functions on $[a, b]$ with the sup norm. By a well-known theorem (cf., for example, Vidossich $[5]), C([a, b])$ is 
separable. Therefore, also $S$ is separable: let $D=\left\{u_{n} \mid n \in \mathbf{N}\right\}$ be a countable dense subset of $S$. Define $\left(v_{n}\right)_{n}$ inductively as follows: $v_{1}=u_{1}$, and $v_{n}$ $=\max \left\{v_{n-1}, u_{n}\right\}$. By the above lemma, $v_{n} \in S$. By construction, $\left(v_{n}\right)_{n}$ is increasing and bounded above by $c$. Therefore, $\left(v_{n}\right)_{n}$ converges pointwise to a bounded, real-valued function $u_{M}$. By $v_{1} \leqq v_{n} \leqq u_{M}$ for all $n$, hypothesis $\left(\mathrm{C}_{3}\right)$ implies the existence of $\left.\left.h \in L_{\mathrm{loc}}^{1}(] a, b\right], \mathbf{R}^{+}\right)$such that $\int_{a}^{b} h(s) d s$ $<+\infty$ and $\left|f\left(t, v_{n}(t)\right)\right| \leqq h(t)\left(a<t \leqq b ; n \in \mathbf{Z}^{+}\right)$. We have

$$
\left|v_{n}(t)-v_{n}(s)\right| \leqq \int_{s}^{t}\left|f\left(\xi, v_{n}(\xi)\right)\right| d \xi \leqq \int_{s}^{t} h(\xi) d \xi,
$$

which implies the equicontinuity of $\left(v_{n}\right)_{n}$ at every point of $[a, b]$ (for the point $a$, it is enough to take $\lim _{s \downarrow a}$ in both sides of the above inequality). Therefore $u_{M}$ is continuous and, hence,

$$
\lim _{t \downarrow a} u_{M}(t)=u_{M}(a)=\lim _{n} v_{n}(a)=x_{0} .
$$

Moreover, $\lim _{n} v_{n}=u_{M}$ uniformly by the Dini theorem. It follows that $u_{M} \in S$ ( $A$ being a closed subset of $\mathbf{R}$ ). To show that $u_{M}$ is the maximal solution, choose $u \in S$. Since $D$ is dense in $S$, there is a sequence $\left(z_{k}\right)_{k}$ in $D$ converging uniformly to $u$. For every $k \in \mathbf{Z}^{+}$, let $n_{k} \in \mathbf{Z}^{+}$be such that $z_{k}=u_{n_{k}}$. From the definition of the $v_{n}$ 's we have $z_{k} \leqq v_{n_{k}} \leqq u_{M}$ (all $k$ ) and, hence, $u \leqq u_{M}$. Q.E.D.

Corollary. Let $A \subseteq \mathbf{R}$ be closed and let $f:[a,+\infty[\times A \rightarrow \mathbf{R}$ satisfy the generalized Carathéodory hypotheses. For the terminal value problem,

$$
x^{\prime}=f(t, x), \quad \lim _{t \uparrow+\infty} x(t)=x_{\infty},
$$

there exists the maximal (resp., the minimal) solution in $[a,+\infty[$ if and only if there exists at least one solution in $[a,+\infty[$ and all solutions are bounded above (resp., below) by a constant $c$.

Proof. Let $\alpha=a-1$. Define $g:] \alpha, a] \times A \rightarrow \mathbf{R}$ by

$$
g(t, x)=-(t-\alpha)^{-2} f\left(\alpha+(t-\alpha)^{-1}, x\right) .
$$

If $x$ is a solution of

$$
x^{\prime}=f(t, x) \text { in }\left[a,+\infty\left[, \quad \lim _{t \uparrow \infty} x(t)=x_{\infty},\right.\right.
$$

then $\bar{x}(t)=x\left(\alpha+(t-\alpha)^{-1}\right), \alpha<t \leqq a$, is a solution of

$$
\left.\left.y^{\prime}=g(t, y) \text { in }\right] \alpha, a\right], \quad \lim _{t \downarrow \alpha} y(t)=x_{\infty} .
$$

If $y$ is a solution of (2), then $\tilde{y}(t)=y\left(\alpha+(t-\alpha)^{-1}\right), t \geqq a$, is a solution of (1). Therefore, we have only to apply Theorem 1 to (2). Q.E.D.

REMARKS. (i) When $A \subseteq \mathbf{R}$ is a right-open interval and $f: I \times A \rightarrow \mathbf{R}$ is continuous, then, using the above results in connection with Theorem 2 of Vidossich [6] for the Cauchy problem and Theorem 1 of [6] for the terminal value problem, we have the following proposition: 
If the maximal solution in I for a given initial or terminal value $x_{0}$ exists, then the maximal solution also exists for every $y_{0} \leqq x_{0}$ provided that there exists at least one solution on I. An analogous result holds for the minimal solution.

It follows that all the well-known results on the existence of global solutions also imply the existence of the maximal solutions.

(ii) It is easy to deduce Theorem 1 of Hallam [1] under the generalized Carathéodory hypotheses from the above corollary, while the well-known result on the existence of local maximal and minimal solutions for the Cauchy problem is a direct consequence of Theorem 1 (and, hence, it can be proved without any comparison or differential inequalities technique). Moreover, for the terminal value problem, we have from the above corollary the existence of the local maximal and minimal solutions under the generalized Carathéodory hypotheses, which seems to be a new result.

\section{On the connectedness of the set of solutions.}

TheOREM 2. Let $A \subseteq \mathbf{R}$ be any interval and let $f:] a, b] \times A \rightarrow \mathbf{R}$ satisfy the generalized Carathéodory hypotheses. For every $x_{0} \in \mathbf{R}$, the set $S$ of solutions in ]a,b] of the limit Cauchy problem (C) is connected for the topology of uniform convergence in $] a, b]$.

Proof. Assume $S \neq \varnothing$, since otherwise the result is obvious. Since every $u$ $\in S$ has a continuous extension on $[a, b]$, we consider $S$ as a subset of the space $C([a, b])$ of all continuous maps $[a, b] \rightarrow \mathbf{R}$ with the sup norm. Suppose $S$ disconnected. Then there exist two nonempty disjoint open sets $U_{1}, U_{2}$ of $S$ $\subseteq C([a, b])$ whose union is $S$. Pick $x_{i} \in U_{i}$ for $i=1,2$. Let $y=\max \left\{x_{1}, x_{2}\right\}$. We shall prove below that the set $S^{*}=\{u \in S \mid u \leqq y$ in $[a, b]\}$ is a connected subset of $C([a, b])$. As $x_{i} \in U_{i} \cap S^{*}, U_{1} \cap S^{*}$ and $U_{2} \cap S^{*}$ are two nonempty disjoint open sets of $S^{*} \subseteq C([a, b])$ whose union is $S^{*}$, a fact which contradicts the claimed connectedness of $S^{*}$. Therefore, such $U_{1}$ and $U_{2}$ cannot exist, and $S$ is connected.

Now we show the connectedness of $S^{*}$. By the above lemma, $y \in S$ and, hence, $y \in S^{*}$. By a well-known theorem (cf., for example, Vidossich [5]), $C([a, b])$ is separable and, hence, $S^{*}$ is too. Let $\left\{u_{n} \mid n \in \mathbf{Z}^{+}\right\}$be a countable dense subset of $S^{*}$. Define $f_{n}:[a, b] \times \mathbf{R} \rightarrow \mathbf{R}$ by

$$
\begin{aligned}
f_{n}(t, x) & =f(t, y(t)) & & \text { if } x>y(t), a<t \leqq b, \\
& =f(t, x) & & \text { if } u_{n}(t) \leqq x \leqq y(t), a<t \leqq b, \\
& =f\left(t, u_{n}(t)\right) & & \text { if } x<u_{n}(t), a<t \leqq b, \\
& =0 & & \text { if } t=a, x \in \mathbf{R} .
\end{aligned}
$$

Obviously $f_{n}$ is bounded and satisfies the generalized Carathéodory hypotheses $\left(f_{n}\right.$ is well defined for $x \in\left[u_{n}(t), y(t)\right]$ since $A$ is an interval). Therefore, the set $S_{n}$ of solutions in $[a, b]$ of the Cauchy problem

$$
x^{\prime}=f_{n}(t, x), \quad x(a)=x_{0}
$$

is nonempty and connected in $C([a, b])$ by a result of Stampacchia [4]. Choose $u \in S_{n}$. If there exists $t_{n} \in[a, b]$ such that $u\left(t_{n}\right)<u_{n}\left(t_{n}\right)$, then let $A_{n}$ 
$=\left\{t \in[a, b] \mid u<u_{n}\right.$ in $\left.\left[t, t_{n}\right]\right\}$ and $\alpha_{n}=\inf A_{n}$. By continuity and $u(a)$ $=u_{n}(a)$, we have $u\left(\alpha_{n}\right)=u_{n}\left(\alpha_{n}\right)$. For almost every $t \in A_{n}, t>\alpha_{n}$, we have

$$
\begin{aligned}
u^{\prime}(t) & =f_{n}(t, u(t)) \\
& =f\left(t, u_{n}(t)\right) \quad\left(\text { by } u \leqq u_{n}\right) \\
& =u_{n}{ }^{\prime}(t) .
\end{aligned}
$$

Taking $\int_{\alpha_{n}}^{t_{n}}$ we get $u\left(t_{n}\right)=u_{n}\left(t_{n}\right)$, a contradiction. Therefore, $u \geqq u_{n}$ in $[a, b]$. In a similar way we prove $u \leqq y$ in $[a, b]$. This means that $u$ belongs to the set $S_{n}^{*}=\left\{v \in S^{*} \mid v \leqq u_{n}\right.$ in $\left.[a, b]\right\}$, and, hence, $S_{n} \subseteq S_{n}^{*}$. Moreover, we obviously have $u_{n}, y \in S_{n}$. Since $y \in \cap_{n=1}^{\infty} S_{n}$, a well-known theorem of General Topology states the connectedness of $E=\cup_{n=1}^{\infty} S_{n}$. Another wellknown theorem of General Topology says that any set included between a connected set and its closure is connected. Therefore, $S^{*}$ is connected since $E$ is dense in $S^{*}$ (by $u_{n} \in E$ ). Q.E.D.

Repeating the argument used to prove the corollary to Theorem 1 and observing that $x \leadsto x \circ \varphi$, where $\varphi(t)=\alpha+(t-\alpha)^{-1}$, is a homeomorphism $C([a,+\infty[) \rightarrow C(] \alpha, a])$, the spaces being endowed with the topology of uniform convergence, we have the following result:

CoROLlaRy. Let $A \subseteq \mathbf{R}$ be any interval and let $f:[a,+\infty[\times A \rightarrow \mathbf{R}$ satisfy the generalized Carathéodory hypotheses. For every $x_{\infty} \in \mathbf{R}$, the set of solutions in $[a,+\infty[$ of the terminal value problem $(\mathrm{T})$ is connected for the topology of uniform convergence in $[a,+\infty[$.

\section{REFERENCES}

1. T. G. Hallam, A comparison principle for terminal value problems in ordinary differential equations, Trans. Amer. Math. Soc. 169 (1972), 49-57. MR 46 \#5733.

2. W. G. Kelley, A Kneser theorem for Volterra integral equations, Proc. Amer. Math. Soc. 40 (1973), 183-190. MR 47 \#5531.

3. S. Nakagiri and H. Murakami, Kneser's property of solution families of non-linear Volterra integral equations, Proc. Japan Acad. Sci. 50 (1974), 296-300.

4. G. Stampacchia, Le transformazioni funzionali che presentano il fenomeno di Peano, Atti Accad. Naz. Lincei Rend. Cl. Sci. Fis. Mat. Nat. (8) 7 (1949), 80-84. MR 11, 442.

5. G. Vidossich, Characterizing separability of function spaces, Invent. Math. 10 (1970), 205-208.

6. Solution of Hallam's problem on the terminal comparison principle for ordinary differential inequalities, Trans. Amer. Math. Soc. (to appear).

Departamento de Matemática, Universidade de Brasília, 70.000 Brasília D. F., Brazil 